\title{
Isotropic-Nematic Phase Separation of a Dispersion of Organophilic Boehmite Rods
}

\author{
P. A. Buining" and H. N. W. Lekkerkerker \\ Van't Hoff Laboratory, University of Utrecht, Padualaan 8, $3584 \mathrm{CH}$ Utrecht, The Netherlands \\ Received: May 7, 1993; In Final Form: August 18, $1993^{\circ}$
}

\begin{abstract}
An isotropic-nematic phase separation is observed in dispersions of sterically stabilized rodlike boehmite particles. The phase separation process is studied with polarization microscopy. Also, the phase volumes and particle concentrations in the coexisting phases are determined. The dependence of these quantities on the total concentration of the dispersion is analyzed in terms of the Onsager theory for the isotropic-nematic phase transition extended to bidisperse mixtures of rodlike particles. Qualitative agreement between theory and experiment is obtained. The triphasic isotropic-nematic-nematic equilibrium as predicted by the theory is observed after 6 months.
\end{abstract}

\section{Introduction}

Dispersions of colloidal particles of rodlike shape may form an ordered nematic phase above a certain particle concentration. The phenomenon of particle alignment has been observed in a wide variety of (aqueous) dispersions of colloidal rodlike particles. As examples, we mention systems of synthetic (hydr)oxidic particles such as $\mathrm{V}_{2} \mathrm{O}_{5},{ }^{1} \beta$-FeOOH, ${ }^{1,2}$ and $\gamma$-AlOOH ${ }^{3,4}$ and organic systems like those of cellulose microcrystals ${ }^{5}$ and poly(tetrafluoroethylene) "whiskers". ${ }^{6}$ An extensively studied, highly monodisperse rod system is that of tobacco mosaic virus (TMV), ${ }^{7}$ which forms ordered phases already at low volume fractions.

The formation of the nematic phase was first explained by Onsager 8 about 50 years ago, who recognized that systems of stiff slender rods may undergo an isotropic-nematic phase separation without attractive interactions between the particles. When the rods become more or less aligned, a loss of orientation entropy is compensated by a decrease in excluded volume. For long rods of length $L$ and diameter $D$, the particle number density where the isotropic-nematic phase coexistence will become stable is determined by their excluded volume (being proportional to $\left.D L^{2}\right)$ :

$$
\rho \propto O(1) / D L^{2}
$$

The corresponding volume fraction of cylindrical rods then shows the proportionality

$$
\phi \propto D^{2} L \rho \propto D / L
$$

So the volume fraction at which phase separation appears is inversely proportional to the aspect ratio of the rods.

Although the direct relevance of the Onsager theory for the explanation of experiments is rather limited being restricted to low volume fractions of perfectly rigid monodisperse very long rods, its extensions by introducing polydispersity and electrostatic interactions and developments for higher volume fractions have been applied with good results to colloidal dispersions (for a recent review see ref 9).

For the TMV system, which shows a hard-core particle interaction with an additional soft repulsion due to electrostatic interaction, Fraden et al. ${ }^{7}$ measured the concentrations of the coexisting isotropic and nematic phases over a wide range of ionic strengths. They found a strong ionic strength dependence of the isotropic-nematic phase boundaries, which clearly demonstrates that the electrostatic repulsion is an important factor in the phase behavior of the system. The theoretical results obtained with the Onsager theory including the effect of screened Coulomb interactions ${ }^{10}$ lie considerably above the experimental

\footnotetext{
- Abstract published in Advance ACS Abstracts, October 1, 1993.
}

values. This is not surprising as the hard-rod dimensions of TMV give a ratio $L / D \approx 17$, which is certainly not large enough for the Onsager approach to be quantitatively valid. Indeed, Sato and Teramoto, ${ }^{11}$ going beyond the second virial approximation for the hard-core part, obtained much better agreement.

This study reports and describes for the first time the isotropicnematic phase transition in a dispersion of organophilic rodlike colloidal particles which can be modeled with a nearly hard particle interaction. The system studied is a dispersion of boehmite particles with an average length of about $200 \mathrm{~nm}$ and an aspect ratio of about 20 , prepared according to a novel alkoxide route. ${ }^{12}$ The boehmite core particles were sterically stabilized by grafting a layer of poly(isobutene) on their surface. ${ }^{13}$

The boehmite dispersion studied here shows polydispersity in both the particle length and width. Nevertheless, the dispersion shows a fast isotropic-nematic phase transition above a certain critical volume fraction. To understand the effect of polydispersity, the coexisting concentrations are compared with those calculated with Onsager's theory extended to a (bidisperse) mixture of two particle lengths. ${ }^{14}$ For disperse solutions, theory predicts the existence of a three-phase isotropic-nematic-nematic region. ${ }^{15-17}$ In bidisperse solutions of the semiflexible polymer Schizophyllan (molecular weight ratio 12), a three-phase coexistence of an isotropic phase with two anisotropic (cholesteric) phases was observed, as well as a biphasic region where two anisotropic phases coexisted. ${ }^{18,19}$ In our case of polydisperse rigid, nearly hard boehmite rods, a second nematic phase indeed emerged in the biphasic dispersion, but not until after months of standing.

\section{Materials and Methods}

The Organophilic Boehmite Dispersion. The dispersion used (coded ASBIP10g) contains boehmite needles, about $200 \mathrm{~nm}$ long ( $\sim 50 \%$ SD), $10 \mathrm{~nm}$ wide ( $\sim 25 \%$ SD), and $8 \mathrm{~nm}$ thick, as determined by transmission electron microscopy. The aqueous dispersion of boehmite core particles was prepared hydrothermally (see ref 12). These particles were stabilized in cyclohexane by terminally grafting low molecular weight poly(isobutene) molecules on their surface, as described in ref 13 . On the basis of the analogy with similar dispersions of spherical silica particles, ${ }^{20}$ we may assume that the grafted rods model a hard interaction. As a consequence of the larger boehmite concentration applied in the grafting procedure compared to that in ref 13, the ASBIP10g dispersion contains a certain amount of clusters consisting of sideways aggregated needles.

A transmission electron micrograph of the dispersion (Figure 1) shows spindlelike bundles of clustered rods next to unclustered rods. It is yet uncertain whether the clusters are reversible aggregates or particle coagulates due to some stickiness of the 


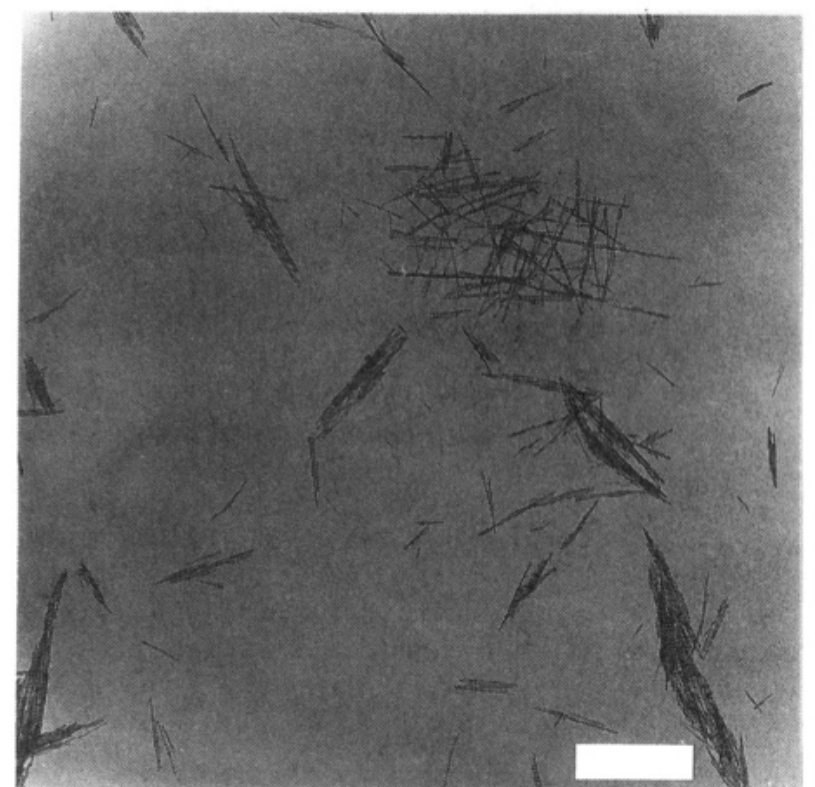

Figure 1. Transmission electron micrograph of ASBIP10g. The length of the bar represents $0.5 \mu \mathrm{m}$.

rods. Elemental analysis of the amounts of carbon $(20.37 \mathrm{wt} \%$; in the polymer chain), nitrogen (1.61 wt \%; in the polymer anchor group), and hydrogen ( $5.44 \mathrm{wt} \%$; in the polymer and core particle) gave a result about identical to that found in ref 13 for a comparable unclustered dispersion. Thus, although particles of ASBIP10g form also clusters, they appear to have a fully grafted surface.

From the elemental analysis and the specific boehmite surface $\left(225 \mathrm{~m}^{2} \mathrm{~g}^{-1}{ }^{13}\right.$ an approximate grafting density of one polymer per $\mathrm{nm}^{2}$ boehmite surface was calculated, which suggests a rather dense polymer packing. The particle density was determined as $2.00 \mathrm{~g} \mathrm{~cm}^{-3}$ by weighing a known volume of dispersion, followed by weighing the solid content after drying the dispersion at 70 ${ }^{\circ} \mathrm{C}$ under a stream of nitrogen gas.

Methods. Starting with a stock ASBIP10g dispersion of $7.36 \%$ $(w / v)$, the concentration was gradually increased by slowly blowing nitrogen through the dispersion under vigorous stirring. The lumps that form during concentration can easily be redispersed by stirring and sonication.

After phase separation, the weight concentration of the isotropic upper phase $\left(w^{i}\right)$ was determined by pipetting off a known volume of upper phase, followed by drying and weighing. The weight concentration of the coexisting nematic lower phase $\left(w^{n}\right)$ was determined from $w^{\mathrm{i}}$, the total concentration of the dispersion $(w)$, and the volumes of the coexisting phases $\left(V^{n}, V^{n}\right)$, using

$$
V^{i} w^{\mathrm{i}}+V^{\mathrm{n}} w^{\mathrm{n}}=\left(V^{\mathrm{i}}+V^{\mathrm{n}}\right) w
$$

Volume fractions were calculated from the expression $\phi^{\mathrm{i}, \mathrm{n}}=w^{\mathrm{i}, \mathrm{n}} /$ $\rho_{\mathrm{p}}$, where $\rho_{\mathrm{p}}$ is the density of the organophilic boehmite particles.

The sample preparation for microscopic study was performed by depositing some dispersion (after being homogenized on a vortex stirrer) into the hollow of a glass slide, onto which a cover slip was put. Soon after preparation the dried dispersion at the borders of the cover slip prevents strong evaporation of the solvent. The sample was observed at a magnification up to $400 \times$ in a Zeiss Axioplan polarization microscope.

\section{Results}

Observations with the Naked Eye. The initial ASBIP10g dispersion $(7.36 \% \mathrm{w} / \mathrm{v})$ appears quite translucent and shows birefringence when the sample tube is shaken. The relaxation time of this shear-induced birefringence is less than $1 \mathrm{~s}$. On increasing the weight concentration above about $9.3 \% \mathrm{w} / \mathrm{v}$, denoted by $w_{\mathrm{i}}$ (corresponding to a volume fraction of $\phi_{\mathrm{i}}=4.7 \mathrm{vol}$
$\%$ ), the dispersion starts to show turbidity, which disappears after shaking and reappears upon resting.

After standing 1 day, the turbid dispersion has separated into a dense phase on the bottom of the tube and a supernatant translucent colloidal dispersion (Figure $2 \mathrm{a}$, for $w=30 \% \mathrm{w} / \mathrm{v}$ ). The lower phase is remarkably transparent and shows a fine structure of permanently birefringent domains, which gradually coarsens with time. The upper phase is isotropic but already displays birefringence when the sample tube is only slightly moved. The interface between both phases is very sharp and moves easily under gravity, which can be observed by tilting the tube (Figure 2b). After being at rest for about 6 months, a thin layer of newly formed birefringent phase was observed on top of the nematic phase (Figure 2c). It appears that during this period the amount of lower nematic remains unchanged. So the new phase originated at the expense of the isotropic upper phase. When the tube is tilted, the layer flows like the lower nematic phase and remains completely separated on top of it.

At a weight concentration of $35.9 \% \mathrm{w} / \mathrm{v}$, denoted by $w_{\mathrm{n}}\left(\phi_{\mathrm{n}}=\right.$ $18.0 \mathrm{vol} \%)$, the point is reached where the isotropic upper phase becomes unstable and the whole dispersion volume will be filled by the nematic phase. Above $w_{\mathrm{n}}$, say at about $50 \% \mathrm{w} / \mathrm{v}(\phi \approx 25$ vol \%), the monophasic nematic dispersion quite suddenly turns into a turbid, extremely viscous gel. The particles appear completely immobilized in a glasslike state.

Microscopic Observations. The growth of the nematic phase follows different stages: In the first stage(A) there is the formation of small ( $\sim 2-4 \mu \mathrm{m})$ birefringent elongated droplets (tactoids), which grow in the isotropic phase (atactosol), as shown in Figure 3a. The large amount of droplets throughout the sample gives it visually a turbid appearance. After a few minutes (stage B), while the droplets are still nucleating, an irregularly structured continuous birefringent phase (tactosol) arises at the bottom of the sample (Figure 3b). During the next few hours (stage $C$ ) the tactoids keep on growing until they are about $30 \mu \mathrm{m}$ in size (Figure $3 c$ ). Some tactoids flow together (Figure 3c, upper right part), and while they become larger, they sink under gravity and merge into the lower tactosol. Often, tactoids appear to nucleate and grow on the lower side of the glass cover slip. When such a tactoid has gained too much weight, a new droplet forms under the old one, connected to it by a thin "neck", recognized as a small dot in the tactoid. Then suddenly the lower tactoid separates from the upper one (the neck disappears and falls apart into small droplets) and starts to sink. The remaining part of the tactoid on the cover slip again grows, and the whole process repeats itself.

As was indicated by Bernal and Fankuchen ${ }^{21}$ by observation of tactoids in aqueous TMV dispersions, the spindlelike shape of a nematic droplet is caused by a balance between surface tension and elastic energy. Under the influence of the tension of the droplet/atactosol interface, the droplet will tend to become spherical. The nematic particle alignment counteracts the formation of a rounded surface by its elasticity. As a compromise, the droplets develop a spindle shape.

In the presence of air bubbles, the tactoids are lined up parallel to the air-sol interface. A special case is formed by domains of atactosol (atactoids), similarly spindle-shaped as the tactoids, surrounded by tactosol (Figure 3c, lower right part). From the settling velocity and the optical contrast of the tactoids, it can be concluded that they have a markedly higher density and refractive index than the surrounding atactosol. During this tactoid growth, the structure of the underlying tactosol (as observed between crossed polars) coarsens and shows the beginning of a schlieren pattern. After 1 day of standing at rest (stage D), all tactoids have disappeared from the isotropic upper phase and the nematic lower phase exhibits a distinct schlieren texture (Figure 3d): a pattern of threadlike brushes which extinguish between crossed polars. The brushes branch out from singular points, which are 

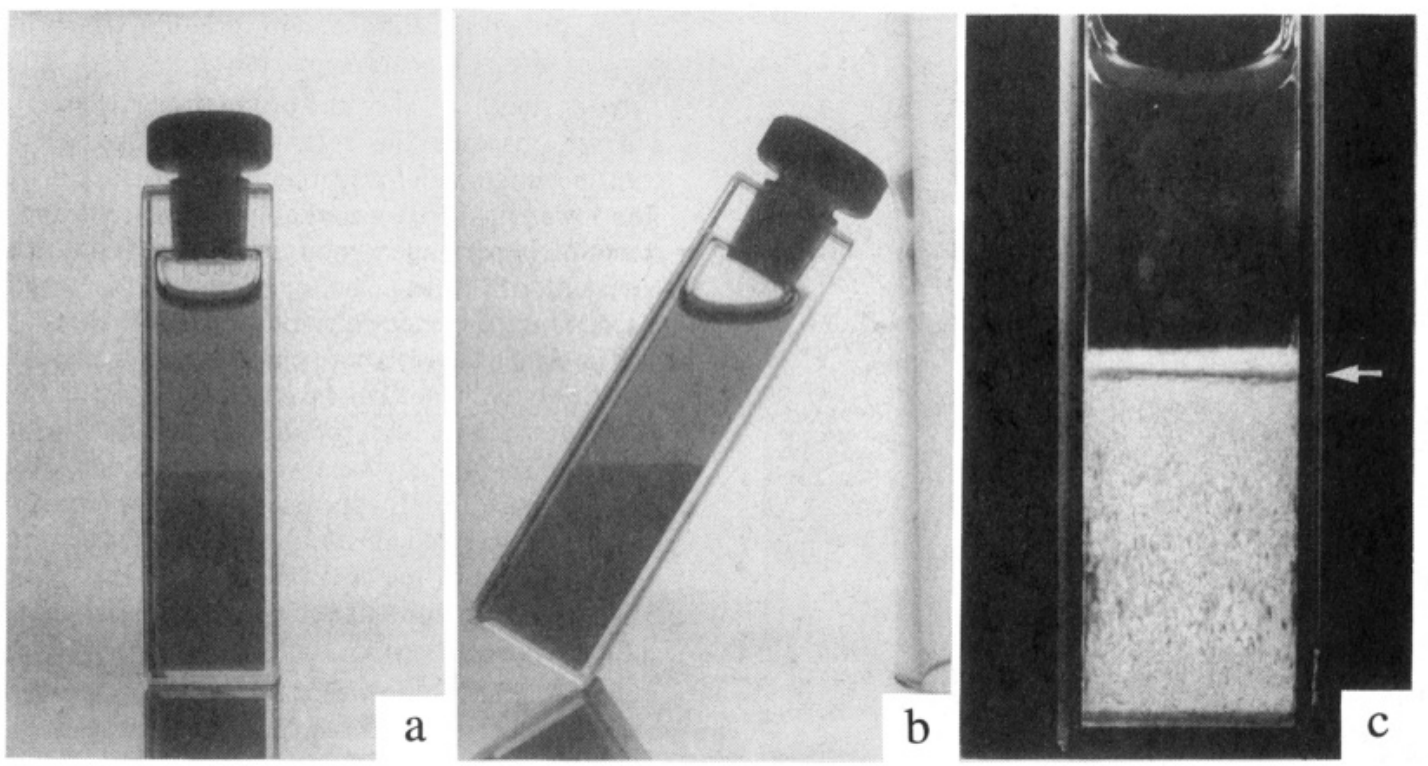

Figure 2. (a) Phase separation of ASBIP10g ( 30\% w/v); (b) $10 \mathrm{~s}$ after tilting the tube; (c) after 6 months, between crossed polars.
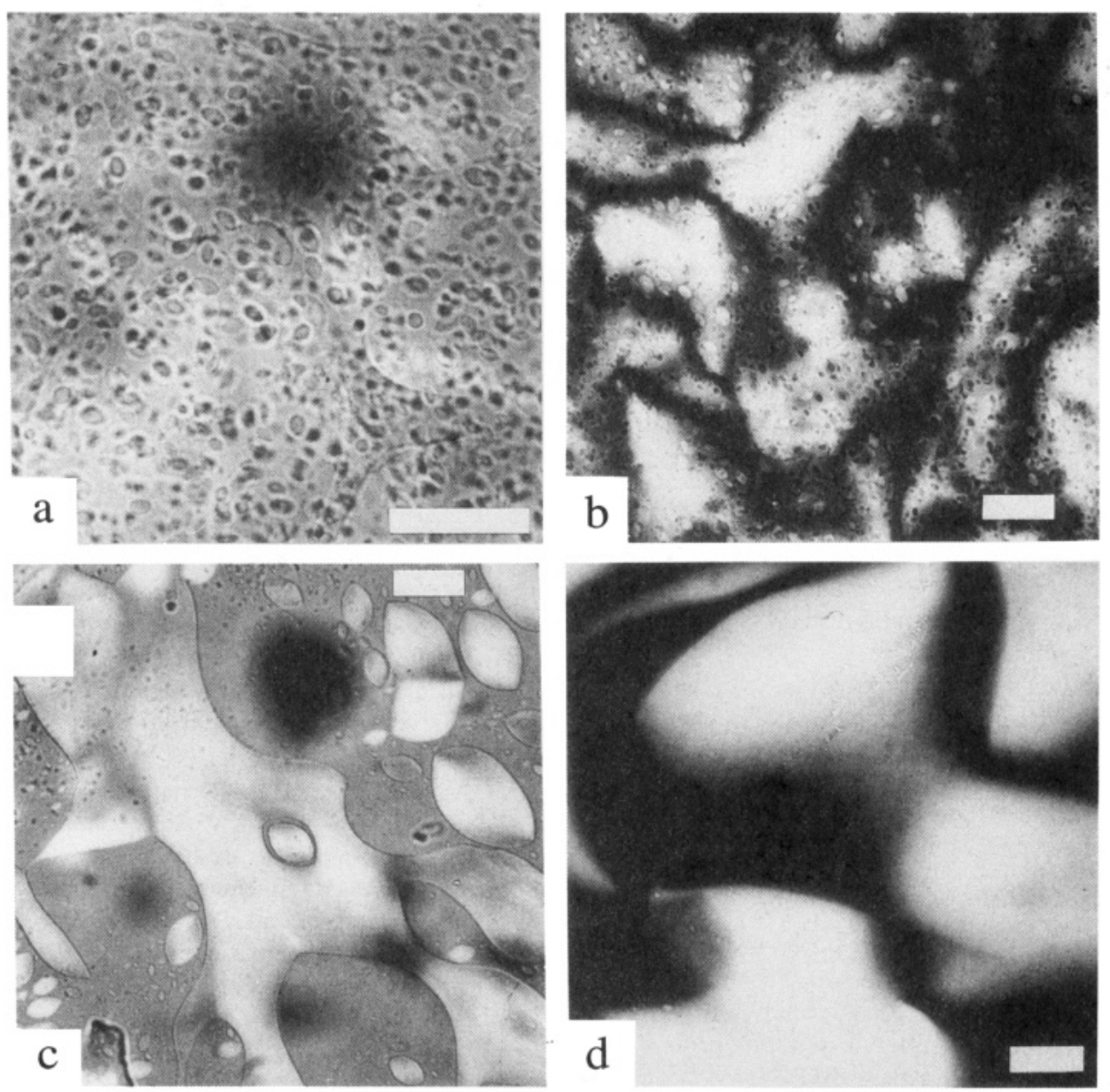

Figure 3. (a) Optical micrograph of the nucleation of very small nematic droplets (stage A). (b) Nematic droplets and underneath a continuous nematic lower phase with schlieren (stage B). (c) Grown-out tactoids (stage C). (d) Schlieren texture after completion of phase separation (stage D). All observations with crossed polars. The lengths of the bars represent $20 \mu \mathrm{m}$.

discontinuities in the mean alignment direction of the particles (referred to as the director). ${ }^{22}$ Around the singular points, the director changes continuously. As a consequence, when the object table is rotated, a singular point stays at a fixed position, while the dark brushes move around it. The schlieren texture resembles the defect structures observed in conventional thermotropic liquid crystals and appears to be extremely sensitive to small disturbances. If the cover slip is shifted slightly, the whole texture changes immediately. The schlieren texture is distorted, and new tactoids are being formed.

Phase Diagram. The experimentally determined boehmite volume fractions of the coexisting isotropic and nematic phases clearly depend on the total volume fraction (Figure 4a). As a consequence, the amounts of nematic phase at several total volume fractions show a nonlinear trend (Figure $4 \mathrm{~b}$ ). For the monodisperse case, one would expect constant volume fractions and a 

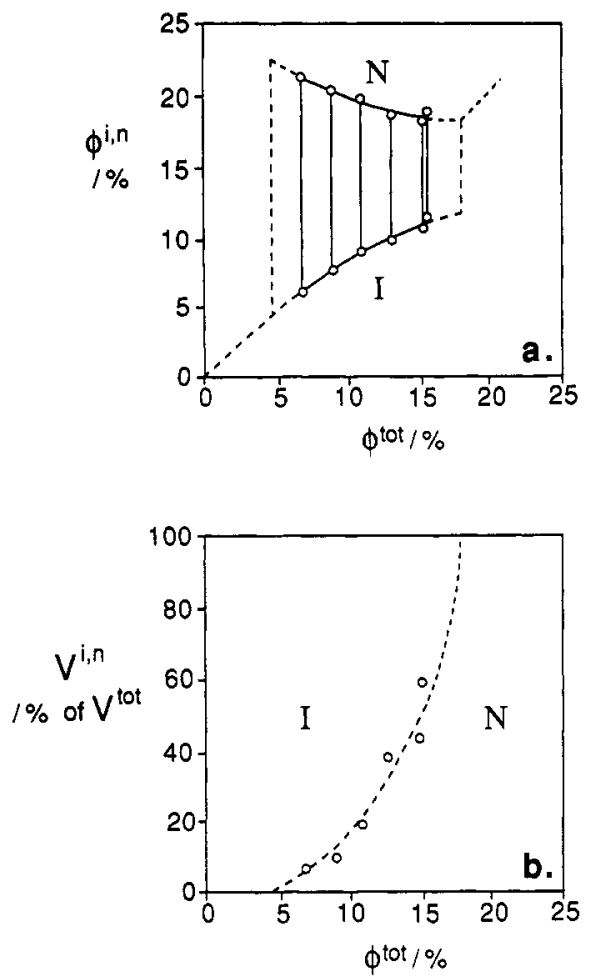

Figure 4. (a) Boehmite volume fractions of coexisting isotropic (I) and nematic (N) phases as a function of the total boehmite volume fraction, and the constructed phase diagram. (b) Volumes of nematic phase (in percentage of total dispersion volume) as a function of the total boehmite volume fraction.

linearly increasing amount of nematic phase in the biphasic region. It is expected that the experimentally found trends find their origin in the system's polydispersity, causing a fractionation effect in the rod length distribution between the isotropic and anisotropic phases. However, visual inspection of the transmission electron micrographs taken of the isotropic and nematic phases which separated in the ASBIP10g dispersion $(12.3 \% \mathrm{w} / \mathrm{v})$ reveals no difference in average particle length or width.

Because theory is available for a bidisperse mixture of rod lengths, we will compare the trends in our experimental phase diagram for the polydisperse case with those in theoretical phase diagrams for the bidisperse case. In the next section we therefore first recapitulate Onsager's theory extended to bidisperse mixtures and then give the phase diagrams for two bidisperse length ratios.

\section{Theory of the Isotropic-Nematic Phase Separation of a Solution of Bidisperse Hard Rods}

In the limit of very long and thin hard rods, the theory of Onsager ${ }^{8}$ for the isotropic-nematic phase separation allows one to make quantitative predictions for the concentrations of the coexisting phases as a function of the length-to-diameter ratio. Onsager treated the isotropic-nematic transition within a virial expansion of the free energy. For very long and thin hard particles the transition occurs at a very low volume fraction, and the virial series may be truncated after the second term. For shorter particles the second virial approximation may not be valid.

Even although in the present case our particles are assumed to approximate closely hard rods, they are polydisperse in length and width. Therefore, the original theory of Onsager cannot be used directly to interpret the experimental data. However, the results of the extension of the Onsager theory to rodlike particles which are polydisperse in length can be used to understand qualitatively the trends in the volumes and concentrations of the coexisting phases.

In a polydisperse system of $N$ particles with equal diameters $D$ and different lengths $L_{j}$, the mole fractions of species $j$ are

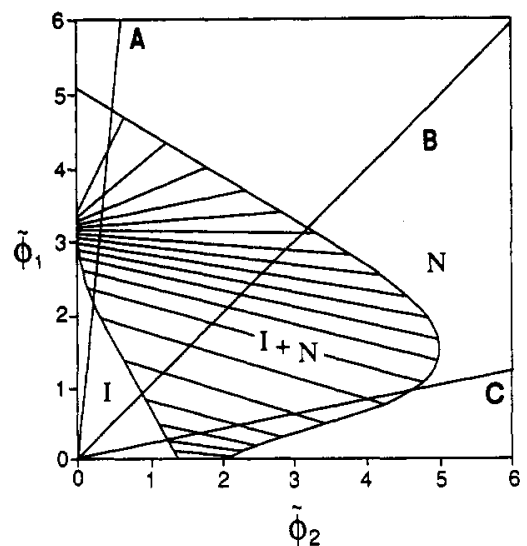

Figure 5. Phase diagram for the system $L_{2} / L_{1}=2.5$, based on calculations using the Gaussian trial function. Volume fractions scaled as $\bar{\phi}=\left(L_{1} /\right.$ D) $\phi$. I, isotropic; $\mathbf{N}$, nematic. Coexisting phases connected by tie lines. Lines $A\left(\phi_{1} / \phi_{2}=10\right), \mathbf{B}\left(\phi_{1} / \phi_{2}=1\right)$, and $\mathbf{C}\left(\phi_{1} / \phi_{2}=0.2\right)$ are profiles of increasing $\phi^{\text {tot }}$

given by $x_{j}=N_{j} / N$, with $N_{j}$ the number of particles of length $L_{j}\left(N=\sum_{j} N_{j}\right)$. The expression for the free energy $\Delta F=$ $F$ (solution) - $F$ (solvent) of the rod system extended to the polydisperse case is given by ${ }^{9}$

$$
\begin{array}{r}
\Delta F / N k T \simeq \text { constant }+\ln c+\sum_{J} x_{j} \ln x_{j}+\sum_{j} x_{j} \sigma_{j}+ \\
c \sum_{j} \sum_{k} x_{j} x_{k} q_{j} q_{k} \rho_{j k}
\end{array}
$$

The dimensionless concentration $c$ is expressed by $c=(\pi / 4)$. $L_{1}^{2} D N / V$, with $(\pi / 4) L_{1}^{2} D$ the (isotropic) excluded volume per shortest rod of length $L_{1}$. The third term $\sum_{\rho} x_{j} \ln x_{j}$ is an entropy of mixing. The fourth term with

$$
\sigma_{j}=\int f_{j}(\Omega) \ln \left[4 \pi f_{j}(\Omega)\right] \mathrm{d} \Omega
$$

is an orientational entropy term. The last term with $q_{j}=L_{j} / L_{1}$ and

$$
\rho_{j k}=(4 / \pi) \iint\left|\sin \gamma\left(\Omega, \Omega^{\prime}\right)\right| f_{j}(\Omega) f_{k}\left(\Omega^{\prime}\right) \mathrm{d} \Omega \mathrm{d} \Omega^{\prime}
$$

expresses the excluded-volume effect on the entropy of the system, which therefore represents a packing entropy term. The angle $\gamma\left(\Omega, \Omega^{\prime}\right)$ is the mutual angle of rods $j$ and $k$ with solid angles $\Omega$ and $\Omega^{\prime}$ with respect to the director. $f_{f}(\theta)$ is the orientation distribution function which gives the probability of a rod of length $L_{j}$ having orientation $\theta$.

The concentrations of rods of length $L_{j}$ in the coexisting isotropic and nematic phases follow from the equalization of the chemical potentials $\left(\mu_{j}=\left[\partial \Delta F / \partial N_{j}\right]_{T, V, N_{i-j}}\right)$ and the osmotic pressures $\left(\Pi_{j}\right.$ $\left.=-[\partial \Delta F / \partial V]_{T, N_{1}}\right)$ of the coexisting isotropic and nematic phases. For the isotropic phase $f_{j}(\theta)=1 / 4 \pi, \sigma_{j}=0$ and $\rho_{j k}=1$. For the nematic phase the free energy is minimized with respect to variations in the orientation distribution functions $f_{j}(\theta)$.

For the bidisperse case of rods of length $L_{1}$ and $L_{2}\left(L_{1}<L_{2}\right)$, Lekkerkerker et al. ${ }^{14}$ performed numerical calculations of the concentrations of short and long rods in the coexisting isotropic and nematic phases for $L_{2} / L_{1}=2$ and 5 . They thereby used an expansion in Legendre polynomials in obtaining expressions for the orientation distribution functions $f_{1}(\theta)$ and $f_{2}(\theta)$. They found a strong fractionation effect (the longer rods going preferentially into the nematic phase) and a widening of the biphasic gap. Odijk and Lekkerkerker ${ }^{23}$ solved the equations analytically using Gaussian distribution functions. By applying the numerical method of Lekkerkerker et al., Birshtein et al. ${ }^{16}$ found triphasic isotropic-nematic-nematic and biphasic nematic-nematic equilibria in a bidisperse system with a $L_{2} / L_{1}$ ratio of 5 . More recently, Vroege and Lekkerkerker ${ }^{17}$ showed that, within the approximation of Gaussian trial functions, the appearance of a second nematic 

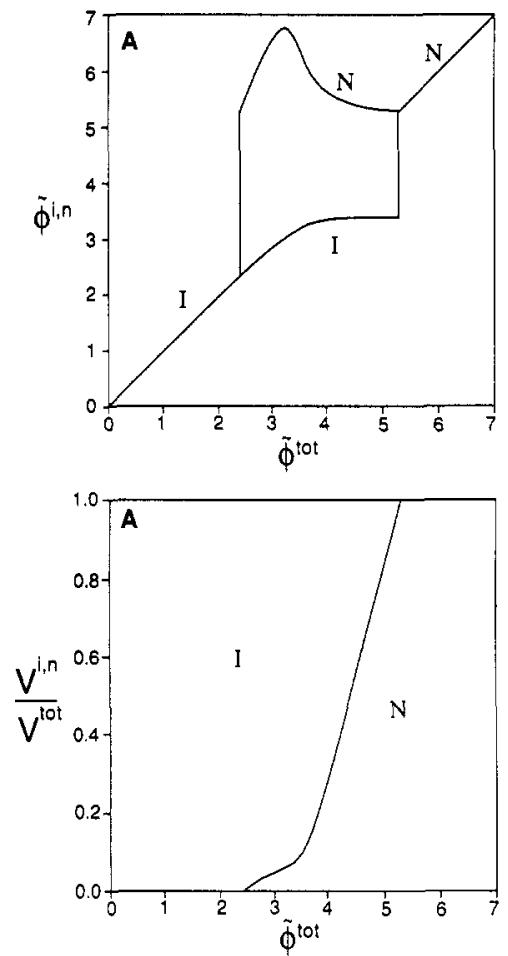
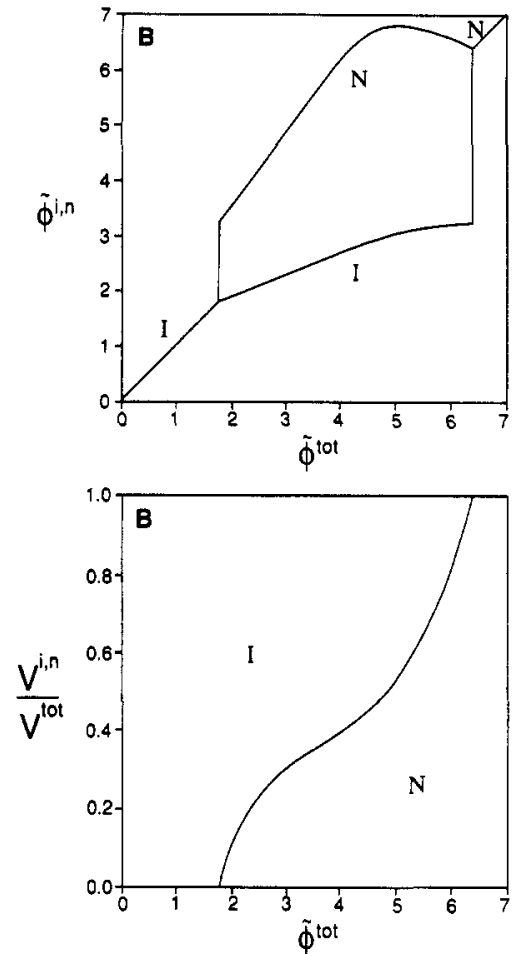
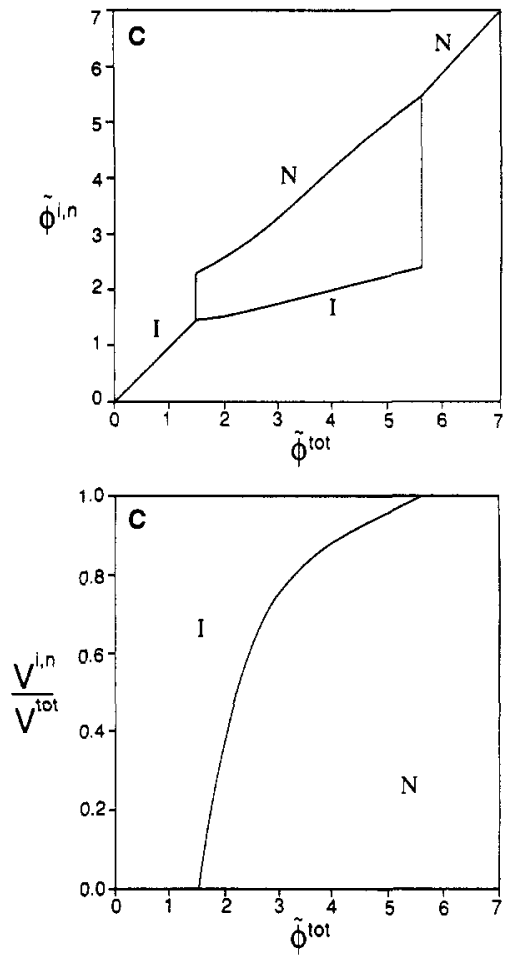

Figure 6. Upper plots: scaled volume fractions of coexisting isotropic and nematic phases in the system $L_{2} / L_{1}=2.5$, as constructed from the tie lines for concentration profiles $\mathrm{A}, \mathrm{B}$, and $\mathrm{C}$ in Figure 5 . Lower plots: the relative amounts of isotropic and nematic phase for profiles $\mathrm{A}, \mathrm{B}$, and $\mathrm{C}$ in Figure 5, calculated according to eq 8 . (A) $\phi_{1} / \phi_{2}=10 ;$ (B) $\phi_{1} / \phi_{2}=1$; (C) $\phi_{1} / \phi_{2}=0.2$.

phase is restricted to a $L_{2} / L_{1}$ ratio of 3.167 and higher. Furthermore, they stated that the nematic-nematic transition is not caused by excluded-volume effects but by a balance between orientational entropy and entropy of mixing.

In this study we will assume the orientation distribution functions to be Gaussian and give phase diagrams of the coexisting concentrations for two $L_{2} / L_{1}$ ratios, namely 2.5 and 3.5 , where the $L_{2} / L_{1}=3.5$ case (taken from ref 17 ) contains a triphasic area. From the calculated values for the mole fractions of long rods $\left(x^{i, a}\right)$ and the dimensionless particle concentrations $\left(c^{i, a}=\right.$ $\left.(\pi / 4) L_{1}^{2} D N^{i, a} / V\right)$ in the isotropic and anisotropic phases, the coexisting (scaled) volume fractions can be determined according to

$$
\tilde{\phi}_{1}^{\mathrm{i}, \mathrm{a}}=\left(1-x^{\mathrm{i}, \mathrm{a}}\right) c^{\mathrm{i}, \mathrm{a}}, \quad \tilde{\phi}_{2}^{\mathrm{i}, \mathrm{a}}=\left(L_{2} / L_{1}\right) x^{\mathrm{i}, \mathrm{a} a} c^{\mathrm{i}, \mathrm{a}}
$$

where $\tilde{\phi}^{\mathrm{i}, \mathrm{a}}=\left(L_{l} / D\right) \phi^{\mathrm{i}, \mathrm{a}}$ with $\phi^{\mathrm{i}, \mathrm{a}}$ the physical volume fraction.

The results for $L_{2} / L_{1}=2.5$ and $L_{2} / L_{1}=3.5$ are shown in Figures 5-8. Figures 5 and 7 show the results plotted for both $L_{2} / L_{1}$ ratios as $\tilde{\phi}_{1}$,i, versus $\tilde{\phi}_{2}$,a, for the coexisting isotropic and anisotropic phases, connected by tie lines. The lines A, B, and $C$ in these plots are lines of increasing $\tilde{\phi}^{\text {tot }}\left(=\tilde{\phi}_{1}+\tilde{\phi}_{2}\right)$ for the dispersion as a whole for different $\phi_{1} / \phi_{2}$ ratios. Experimentally, these lines represent the phase behavior upon evaporating the solvent from the bidisperse mixture. For each of these lines, the volume fractions of the coexisting isotropic and anisotropic phases $\left(\bar{\phi}^{i, a}=\left(\bar{\phi}_{1}+\bar{\phi}_{2}\right)^{i, a}\right)$ are determined by the tie lines as a function of $\tilde{\phi}^{\text {tot }}$, as presented in the upper plots in Figure $6 \mathrm{~A}-\mathrm{C}\left(L_{2} / L_{1}=\right.$ $2.5)$ and figure $8 \mathrm{~A}, \mathrm{~B}\left(L_{2} / L_{1}=3.5\right)$. The fraction of volume of phase $\alpha$ in the biphasic $\alpha-\beta$ case at a certain $\tilde{\phi}^{\text {tot }}$ value is determined from $\tilde{\phi}_{1,2^{\alpha}}$ and $\tilde{\phi}_{1,2^{\beta}}$ using

$$
y^{\alpha} \tilde{\phi}_{j}^{\alpha}+\left(1-y^{\alpha}\right) \tilde{\phi}_{j}^{\beta}=\tilde{\phi}_{j}^{\text {tot }} \quad \text { with } j=1 \text { or } 2
$$

with $y^{\alpha}=V^{\alpha} / V^{\text {not }}$. For the triphasic $\alpha-\beta-\gamma$ case we use

$y^{\alpha} \tilde{\phi}_{j}^{\alpha}+y^{\beta} \tilde{\phi}_{j}^{\beta}+\left(1-y^{\alpha}-y^{\beta}\right) \tilde{\phi}_{j}^{\gamma}=\tilde{\phi}_{j}^{\text {tot }} \quad$ with $j=1$ and 2

For the $L_{2} / L_{1}$ and $\phi_{1} / \phi_{2}$ ratios mentioned above the volumes are shown in the lower plots in Figures 6 and 8.

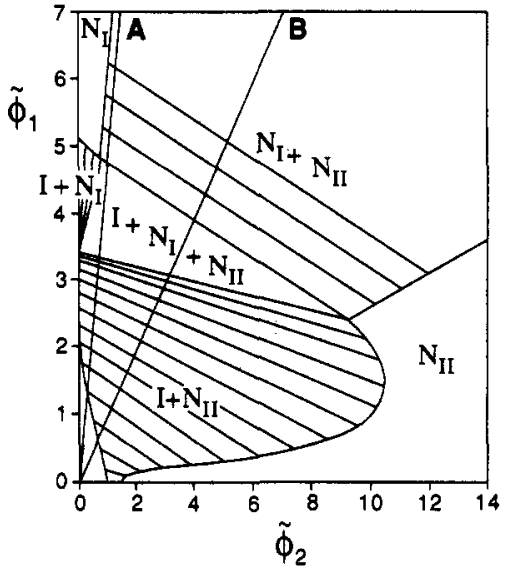

Figure 7. Phase diagram of scaled volume fractions $\left(\tilde{\phi}=\left(L_{1} / D\right) \phi\right)$ for the system $L_{2} / L_{1}=3.5$ taken from ref 17 (Gaussian trial function). I, isotropic; $\mathbf{N}_{\mathbf{l}}, \mathbf{N}_{\mathrm{II}}$, nematic. Coexisting phases in the biphasic regions connected by tie lines. Lines $A\left(\phi_{1} / \phi_{2}=5\right)$ and $B\left(\phi_{1} / \phi_{2}=1\right)$ are profiles of increasing $\bar{\phi}^{\text {tot }}$.

\section{Discussion of the Experimental and Theoretical Phase Diagrams}

Comparison of the experimental phase diagram (Figure 4) with the theoretical curves (Figures 6 and 8 ) shows that the experimental findings agree most well with the case of $L_{2} / L_{1}=$ 2.5 and a large $\phi_{1}\left(\phi_{2}\right.$ ratio (the $A$ line in Figure 5). The maximum in the $\tilde{\phi}^{\mathrm{n}}$ versus $\phi_{\text {tot }}$ plot in Figure $6 \mathrm{~A}$ might also be present on the low volume fraction side of the experimental biphasic area but is difficult to investigate because of the very small amounts of nematic phase. The agreement with case $A$ can be explained from the rod length distribution in the dispersion as follows. It was observed earlier (see refs 24 and 12, histograms and electron micrographs) that dispersions of hydrothermally grown boehmite needles contain a small fraction of very long particles of a length up to about 3 times the average particle length. This would indeed result in a large $\phi_{1} / \phi_{2}$ ratio in the particle length distribution. That the fractionation effect is not visually evident from the 

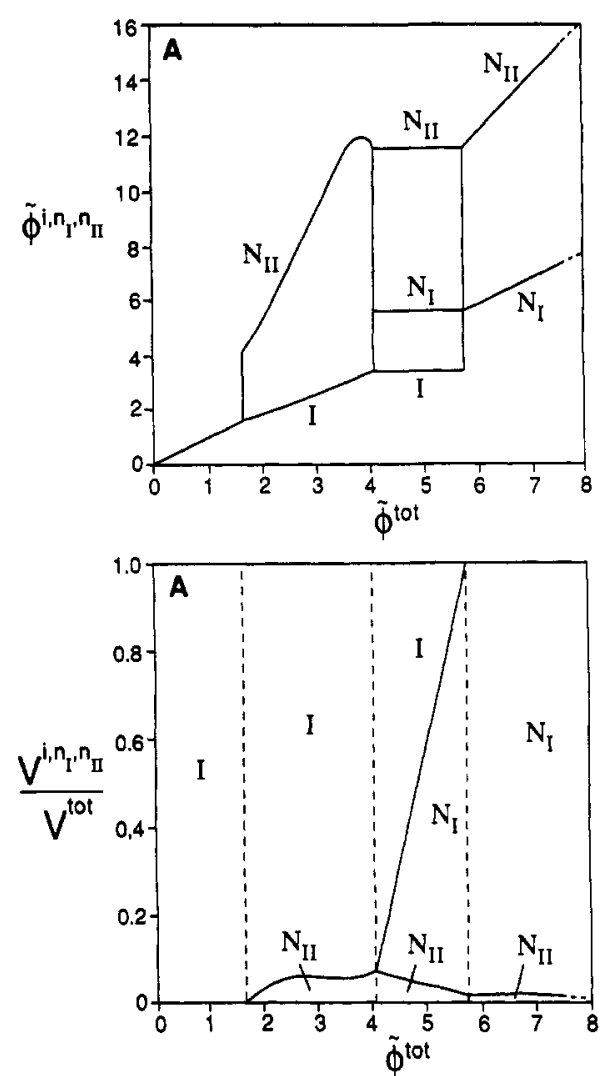
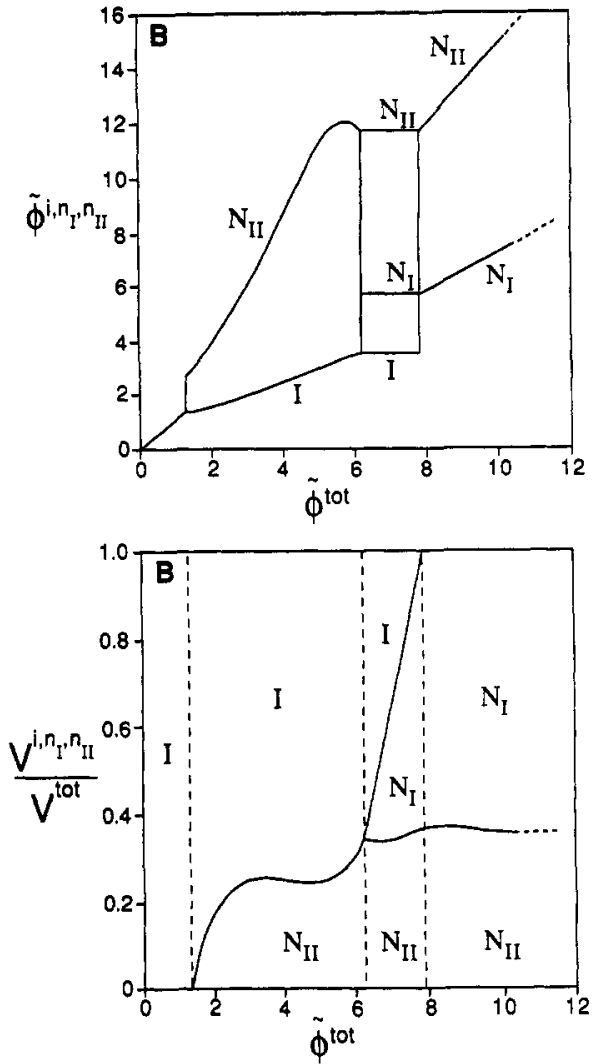

Figure 8. Upper plots: scaled volume fractions of coexisting isotropic and nematic phases in the system $L_{2} / L_{1}=3.5$, as constructed from the tie lines for profiles $A$ and $B$ in Figure 7. Lower plots: the relative amounts of isotropic and nematic phase for profiles $A$ and $B$ in Figure 7 , calculated according to eq 8 (biphasic) and eq 9 (triphasic). (A) $\phi_{1} / \phi_{2}=5 ;(B) \phi_{1} / \phi_{2}=1$.

electron micrographs may be explained by the facts that the system is quite polydisperse and the fractionation is largely determined by the very long rods, which form just a minor amount in the dispersion.

The experimentally determined biphasic gap between $\phi_{i}=4.7 \%$ and $\phi_{\mathrm{n}}=18.0 \%$ yields a $\phi_{\mathrm{n}} / \phi_{\mathrm{i}}$ ratio of 3.8. For the monodisperse case one finds $\phi_{\mathrm{n}} / \phi_{\mathrm{i}}=1.3$ (see ref 14), so the widening of the biphasic gap due to polydispersity is evident. Case $\mathrm{A}$ in Figure $S$ is quite representative for our ASBIP10g system on choosing the following system parameters: $91 \%$ volume fraction of rods with length $L_{1}=200 \mathrm{~nm}$ (average rod length) and $9 \%$ volume fraction of rods with $L_{2}=500 \mathrm{~nm}$ (length of the few very long rods), both having $D=10 \mathrm{~nm}$. The above-mentioned experimental values of $\phi_{\mathrm{i}}$ and $\phi_{n}$ are however lower than the values $\phi_{\mathrm{i}}=12 \%$ $\left(\tilde{\phi}_{i}=2.4\right)$ and $\phi_{n}=27 \%\left(\tilde{\phi}_{n}=5.3\right)$ predicted from Figure 6A. Deviations from the theory are to be expected for the following reasons: (1) the particle aspect ratios in the ASBIP10g system are, strictly speaking, not large enough for the Onsager approach to be quantitatively valid; (2) the particle interaction might not be completely hard but may show some additional attraction or repulsion; ( 3 ) because our system is polydisperse, its modeling by bidispersity might not fully explain the trends; and (4) the theory is based on the Gaussian approximation.

From Figure $7\left(L_{2} / L_{1}=3.5\right)$ the experimentally found triphasic isotropic-nematic-nematic coexistence can be explained. Obviously, the system shows a high enough polydispersity to reach a maximum $L_{2} / L_{1}$ ratio of about 3.5. However, the formation of the second nematic phase, though being the stable configuration, takes a considerable amount of time. So, the biphasic dispersion can be assumed to be metastable and to gradually transform to the triphasic scenario of Figure 8. Comparing Figures $6 \mathrm{~A}$ and $8 \mathrm{~A}$, we would then expect the nematic phase in the biphasic dispersion to separate into two nematic phases. However, we observed that the newly formed nematic phase separated from the isotropic phase.

\section{Concluding Remarks}

For the first time to our knowledge the isotropic-nematic phase separation is observed in a dispersion which is a model for a hard rod system. In this system of sterically stabilized boehmite rods (on average $200 \mathrm{~nm}$ long, $L / D \approx 20$ ), the biphasic gap is situated between $\phi_{\mathrm{i}}=4.7 \%$ and $\phi_{\mathrm{n}}=18.0 \%$. At $\phi \approx 25 \%$ the monophasic nematic transforms into an extremely viscous glass phase.

The nematic phase formation is initiated by the formation of small nematic droplets, to be recognized as an increase in the turbidity of the dispersion. These tactoids slowly settle under gravity to form the continuous nematic phase. The effect of polydispersity on the phase diagram is, given the approximations, well predicted by the Onsager theory for bidisperse rod systems of large $\phi_{1} / \phi_{2}$ ratio. The three-phase isotropic-nematic-nematic equilibrium as predicted by theory is observed. The second nematic phase, which situates itself on top of the lower nematic phase, develops very slowly (period of months!). The reasons for this slow growth are still unclear at this moment.

Acknowledgment. The authors thank Gert-Jan Vroege for critical reading of the manuscript which led to several improvements, for carrying out the numerical calculations necessary for Figure 5, and for providing Figure 7.

\section{References and Notes}

(1) Zocher, H. Z. Anorg. Chem. 1925, 147, 91.

(2) Maeda, Y.; Hachisu, S. Colloids Surf. 1983, 6, 1.

(3) Zocher, H.; Török, C. Kolloid Z. 1960, 170, 140; 1960, 173, 1; 1962 , 180,41

(4) Buining, P. A. Preparation and Properties of Dispersions of Colloidal Boehmite Rods. Ph.D. Thesis, Utrecht University, The Netherlands, 1992.

(5) Marchessault, R. H.; Morehead, F. F.; Walter, N. M. Nature 1959, 184,632 . 55.

(6) Folda, T.; Hoffmann, H.; Chanzy, H.; Smith, P. Nature 1988, 333,

(7) Fraden, S.; Hurd, A. J.; Meyer, R. B.; Cahoon, M.; Caspar, D. L. D. J. Phys. (Paris) 1985, 46C3, 85. 
(8) Onsager, L. Phys. Rev. 1942, 62, 558; Ann. N.Y. Acad. Sci. 1949, 51,627 . 1241

(9) Vroege, G. J.; Lekkerkerker, H. N. W. Rep. Prog. Phys. 1992, 55,

(10) Stroobants, A.; Lekkerkerker, H. N. W.; Odijk, T. Macromolecules 1986, 19, 2232.

(11) Sato, T.; Teramoto, A. Physica A 1991, 176, 72.

(12) Buining, P. A.; Pathmamanoharan, C.; Jansen, J. B. H.; Lekkerkerker, H. N. W. J. Am. Ceram. Soc. 1991, 74, 1303.

(13) Buining, P. A.: Veldhuizen, Y. S. J.; Pathmamanoharan, C. Lekkerkerker, H. N. W. Colloids Surf. 1992, 64, 47.

(14) Lekkerkerker, H. N. W.; Coulon, P.; van der Haegen, R.; Deblieck, R. J. Chem. Phys. 1984, 80, 3427.
(15) Flory, P. J.; Abe, A. Macromolecules 1978, 11, 1119.

(16) Birshtein, T.M.; Kolegov, B. I.; Pryamitsyn, V. A. Vysokomol. Soyed. 1988, A30, 348; Polym. Sci. U.S.S.R. 1988, 30, 316.

(17) Vroege, G. J.; Lekkerkerker, H. N. W. J. Phys. Chem. 1993, 97, 3601 .

(18) Itou, T.; Teramoto, A. Polym. J. 1984, 16, 779.

(19) Sato, T.; Ikeda, N.; Itou, T.; Teramoto, A. Polymer 1989, 30, 311.

(20) Pathmamanoharan, C. Colloids Surf. 1988/89, 34, 81.

(21) Bernal, J. D.; Fankuchen, I. J. Gen. Physiol. 1941, 25, 111.

(22) Friedel, G. Ann. Phys. 1922, 18, 273.

(23) Odijk, T.; Lekkerkerker, H. N. W. J. Phys. Chem. 1985, 89, 2090.

(24) Buining, P. A.; Pathmamanoharan, C.; Bosboom, M.; Jansen, J. B. H.; Lekkerkerker, H. N. W. J. Am. Ceram. Soc. 1990, 73, 2385. 